\title{
On predicting exponents for cross-modality matches
}

\author{
S. S. STEVENS ${ }^{2}$ \\ HARVARD UNIVERSITY
}

\begin{abstract}
Mashhour and Hosman used magnitude estimations to scale seven continua: line longth, time duration, finger span, loudness of noise, weight, gray reflectance, and surface area. The first four continua also served as the adjusted stimuli in 17 cross-modality matches among the various continua. Contrary to the view expressed by Mashhour and Hosman, the results appear to support the psychophysical power law. A reanalysis of the data shows that the exponents of the power functions obtained in cross-modality matches agree with the exponents of the power functions produced by magnitude estimations, provided correction is made for the regression effect. The measured discrepancies hetween the exponents predicted and those actually obtained show scatter that is consistent with that of other experiments. In particular, the scatter accords well with the distribution of 68 exponents predicted by Moskowitz from experiments in which Os matched both number and loudness to various taste concentrations.
\end{abstract}

Since the data of Mashhour and Hosman (1968a) represent an extensive study of cross-modality matching, it appears worthwhile to see in what ways the data may instruct us further regarding the psychophysical law. The authors themselves expressed a pessimistic view. They concluded that "the empirical outcomes of the present investigation speak unfavorably against the validity of the ratio scales and the psychophysical power law ...." and in a fuller version of their report (1968b) they asked, "How can this conclusion invalidate the abundant evidence in support of the power law obtained so far in hundreds of experiments?" The answer, they said, was that those power functions were "obtained on the graph by curve fitting procedures.... In a validation study," they went on to say, "we are primarily concerned with the empirical content of a formula which is to be called a law, its invariance and power of prediction, rather than its appearance on the graph. And it is in this sense that the power function falls short of a natural law."

Nevertheless, the data of Mashhour and Hosman support the power law rather well, at least "on the graph." And when the regression effect is taken into account, the cross-modality matches exhibit the expected transitivity.

\section{THE EXPERIMENTS}

Since details are given by Mashhour and Hosman, the procedure may be briefly summarized.

Twenty Os produced 17 equal-sensation functions by adjusting a stimulus on one continuum to match various levels of a criterion stimulus on another continuum. There were seven continua in all, four of which could be adjusted by the Os. Six of the continua served as the criterion in at least one of the 17 experiments.

After the 17 cross-modality matching functions had been obtained, the Os were asked to match numbers to each of the seven continua, a form of cross-modality matching that is usually called magnitude estimation. The $\mathbf{E}$ designated a standard stimulus, called 10, which was presented before each stimulus level. On each continuum, a different stimulus served as the standard in each of three separate experiments. Thus there were 21 magnitude estimation experiments.

In each experiment five matches were made to each criterion stimulus. The order of the various stimulus levels was irregular. Geometric means were computed across trials and Os. Tables of the geometric means were kindly made available by Dr. Mashhour.

\section{EFFECT OF THE STANDARD}

The effects produced by using a standard at one or another position in the series of stimuli to be estimated was explored in judgments of loudness (Stevens, 1956). Apart from certain standards that were painfully loud, most positions of the standard turned out to have relatively minor effects. There was a tendency for the functions plotted in log-log coordinates to be slightly steeper when the standard was placed near the center of the range than when it was placed near one or the other end of the range. The same effect is detectable in the data of Mashhour and Hosman when the slopes for the three different standards are averaged across six of the continua (omitting the rather variable results for lightness of grays). The average values run: low standard, 1.01; middle standard, 1.13; and high standard, 1.08.

Although the position of the standard exerted an influence on the exponent, the influence was no more than a second-order effect. It appears justifiable, therefore, to average the data obtained with the various standards in order to determine a single magnitude estimation function for each continuum.

As was noted in the study of loudness (Stevers, 1956), the best thing to do with the standard is to omit it. Except in special circumstances, the presentation of a standard and the designation of a modulus have proved to be quite unnecessary. It is especially pointless and wasteful to repeat the standard before each variable stimulus. Nevertheless, it is improbable that the results obtained by Mashhour and Hosman would have been altered to a substantial degree if the Es had saved themselves the time and effort that it costs to administer repeated standards in magnitude estimation experiments.

\section{MAGNITUDE ESTIMATION FUNCTIONS}

Since the position of the standard had no large effect on the magnitude estimations, all the data for a given continuum have been pooled. Geometric means and least-square slopes, in log-log coordinates, were obtained by a local computer program known as PSYCHOFIT. The geometric means are plotted in Fig. 1. The horizontal positions of the functions are arbitrary, since we are concerned here only with the slopes (exponents) of the functions.

It would appear from the overall linear character of the functions in Fig. 1 that the power law is corroborated, at least to a reasonable degree. It should be noted, however, that the function for lifted weight is curved concave downward and the function for lightness of grays is noisy. The reason for the concavity of the weight function is not clear, although the procedure used was unconventional in that the $O$ lifted the weight by a cord. The general noisiness in the judgments of the lightness of gray papers occasions no surprise, for previous Es have failed in their efforts to subdue the noise and variability in lightness judgments (Stevens \& Galanter, 1957; Torgerson, 1960). The results from three different laboratories now show that grayness is a difficult continuum to judge.

The exponents determined by the lines in Fig. 1 are shown in Table 1. Good agreement with the exponents obtained in other 


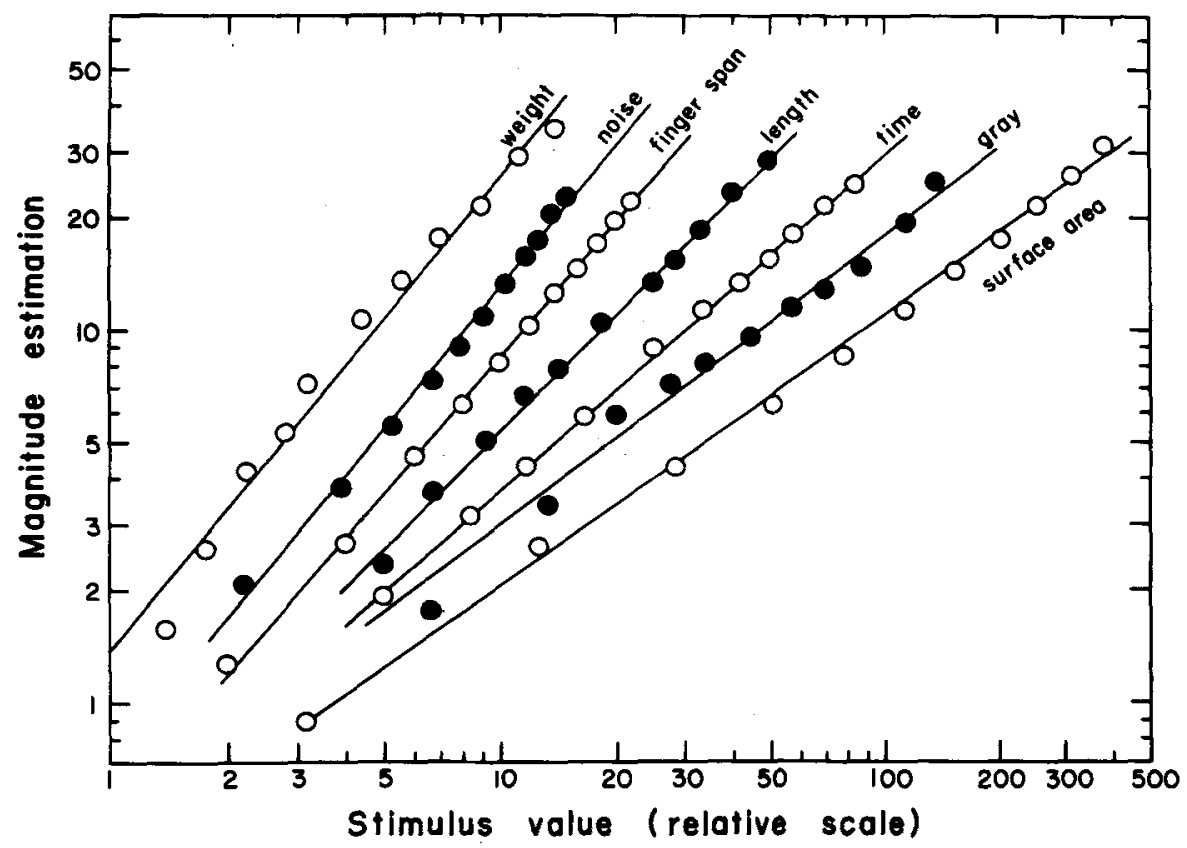

Fig. 1. Psychophysical functions obtained by magnitude estimation. Each point is based on 15 judgments by each of 20 Os. The slopes of the lines, which give the exponents of the functions, were determined by the method of least squares. The horizontal position of each function is arbitrary. laboratories is shown by the magnitude estimations for most of the continua, e.g., finger span, apparent length, apparent duration, and apparent size of surface areas. In addition the exponent for lifted weight agrees quite well with the values reported by Stevens and Galanter (1957), both for an experiment in which a standard was always available and for another experiment in which the standard was presented before every third stimulus to be judged. It should be noted that in this latter experiment each stimulus was judged a total of six times. When that experiment was carried out in 1956 it was not yet known that little or no new information is obtained with magnitude estimation by asking the $O$ s to judge a given stimulus more than once or twice. Mashhour and Hosman seem to think otherwise, for they make a point of the fact that their Os made five judgments per stimulus.

The downward concavity of the function for weight shown in Fig. 1 was not found in the 10 separate estimation experiments reviewed by Stevens and Galanter. On the contrary, there was a slight but general tendency for the estimation judgments to give curves that were concave upward.

A remarkable feature of Table 1 is the size of the noise exponent, which is approximately double the value of the exponent of the standard sone function. What could have produced such an unusual result? There may have occurred an error of some kind, for the authors, in relating sound pressure level to voltage applied to the transducer, give an equation that has in it the rather puzzling term $15.92 \log \mathrm{mV}$. For a linear transducer system the SPL is 20 times the logarithm of the voltage ratio, so we are left with an open question concerning the stimulus values. On the assumption that the stimulus values are correctly stated by Mashhour and Hosman, the total stimulus range was $16.7 \mathrm{~dB}$. That is a very short range. When a similar short range (about $16 \mathrm{~dB}$ ) was used with luminous targets in the dark (see S. S. Stevens \& J. C. Stevens, 1960) it was observed that the brightness function determined by magnitude estimation was fairly close to its normal form after the first series of judgments, but that the brigh tness function became steeper with succeeding presentations of the stimuli. The slope (exponent) was about 0.4 for the first presentation and about 0.6 for the third. Since Mashhour and Hosman used five presentations of each stimulus, the steepening effect caused by the short range was presumably greater than that observed in the brightness study. It would be interesting to know whether the first set of loudness judgments fell closer to the sone scale.

\section{THE ADDITIVE CONSTANT}

When Mashhour and Hosman plotted the data presented in Fig. 1 they plotted a separate function for each of the three standards used with each continuum. And whenever the function appeared curved in the log-log coordinates, they added or subtracted a constant in order to straighten the line on the graph. On a log-log plot the straightening occurs because the additive constant affects the position of the small values more than that of the large values. Six of the 21 functions were subjected to the additive constant. Similarly, since 12 of the 17 cross-modality functions showed some degree of curvature (six of them curved upward, six downward), constants were added or subtracted as needed in order to produce more linear graphs.

Such arbitrary use of an additive constant may mislead, because the constant may alter the exponent of the power function in a way that leads to a misrepresentation. How the additive constant affects the exponent can be illustrated by the three magnitude-estimation functions for loudness shown in the paper by Mashhour and Hosman. Two of the functions appeared reasonably straight, but the third showed an upward concavity due mainly to the position of a single point at the bottom of the function. A constant equal to $\mathbf{0 . 1 4}$ was added to all the measured stimulus values, whereupon the function became straighter and the exponent changed from 1.3 to 1.9. The exponents for the two functions to which constants were not added were 1.3 and 1.1 .

Table 1

Exponents of psychophysical functions determined by magnitude estimation. The values correspond to the slopes of the lines in Fig. 1.

Lifted weight (W)

Loudness of noise (N)

Finger span (F)

Apparent length (L)

Apparent duration (T)

Lightness of grays (G)

Size of surface area (S) 
Since the log-log slope, and hence the size of the exponent, is sensitive to the addition of a constant, the constant ought to be added only when there is a demonstrable necessity for it. The straightening of a particular line is not in itself a sufficient reason for adding a constant. There appear to be only a few circumstances in which the additive constant has a justified rationale. It may serve, for example, as a threshold constant in the description of visual brightness (as affected by adaptation, but not as affected by contrast-J. C. \& S. S. Stevens, 1963). It may also serve to describe the loudness function near threshold, although that is not a settled matter (for a review of the issues, see Marks \& J.C.Stevens, 1968). The functions governing the sensations of tactual warmth, cold, and pain produced by thermal or radiant stimuli apparently have need of an additive constant. Warmth, for example, begins to be felt only when the stimulus temperature exceeds about $305 \mathrm{deg} \mathrm{K}$. If the stimulus is measured as distance above that point, often called physiological zero, the sensation of warmth grows as a power function.

The success of the additive constant in the few places where it has proved helpful does not justify its indiscriminate use. When curvature is accidental or due to a source of error-or when the curvature is not a replicable feature of the psychophysical function in question-under those circumstances it seems wise not to resort to line straightening. In a project like that undertaken by Mashhour and Hosman, where the main experimental query concerns the degree of consistency or transitivity among the exponents, it seems important to avoid the risk of inviting a wrong answer by way of an altered set of exponents. The primary interest concerns the concordance among the exponents before any constants have been added or subtracted.

\section{REGRESSION}

With the additive constants removed, as in Fig. 1, the magnitude estimation functions may be used to predict the results to be expected from cross-modality matching. If there were no systematic errors either in the magnitude estimations or in the cross-modality matches, any two slopes (exponents) in Fig. 1 could be used to predict the exponent of the equal-sensation matching function. The equal-sensation exponent would be given by the ratio between two slopes in Fig. 1. For the 17 cross-modality matching experiments performed by Mashhour

Table 2

Exponents for cross-modality matches in experiments by Mashhour and Hosman. Where appropriate, the exponents predicted from magnitude estimations (Fig. 1 and Table 1) were corrected for the regression effect. The last column gives the difference in decilogs between the predicted or corrected exponents and the obtained exponents.

\begin{tabular}{|c|c|c|c|c|c|}
\hline & & Slope & (exponen & & \\
\hline & & predicted & corr'd for & ob- & Difference in \\
\hline criterion & adjusted & from M.E. & regression & tained & \\
\hline Noise & time & 1.37 & 1.07 & 1.2 & .50 \\
\hline Noise & length & 1.19 & & 1.1 & .34 \\
\hline Noise & finger span & 1.04 & .67 & .72 & .31 \\
\hline Time & length & .87 & & .95 & .38 \\
\hline Time & finger span & .76 & .48 & .48 & 00 \\
\hline Length & finger span & .87 & .55 & $.5 z$ & .24 \\
\hline Weight & noise & 1.04 & & 1.22 & \\
\hline Weight & time & 1.43 & 1.10 & 1.04 & .59 \\
\hline Weight & length & 1.24 & & 1.06 & .68 \\
\hline Gray & noise & .62 & & .91 & \\
\hline Gray & time & .85 & .65 & .75 & .62 \\
\hline Gray & length & .73 & & .83 & .51 \\
\hline Gray & finger span & .64 & .41 & .46 & .50 \\
\hline Surface & noise & .58 & & .63 & \\
\hline Surface & time & .79 & .61 & .56 & .37 \\
\hline Surface & length & .69 & & .55 & .99 \\
\hline Surface & finger span & .60 & .38 & .29 & 1.18 \\
\hline
\end{tabular}

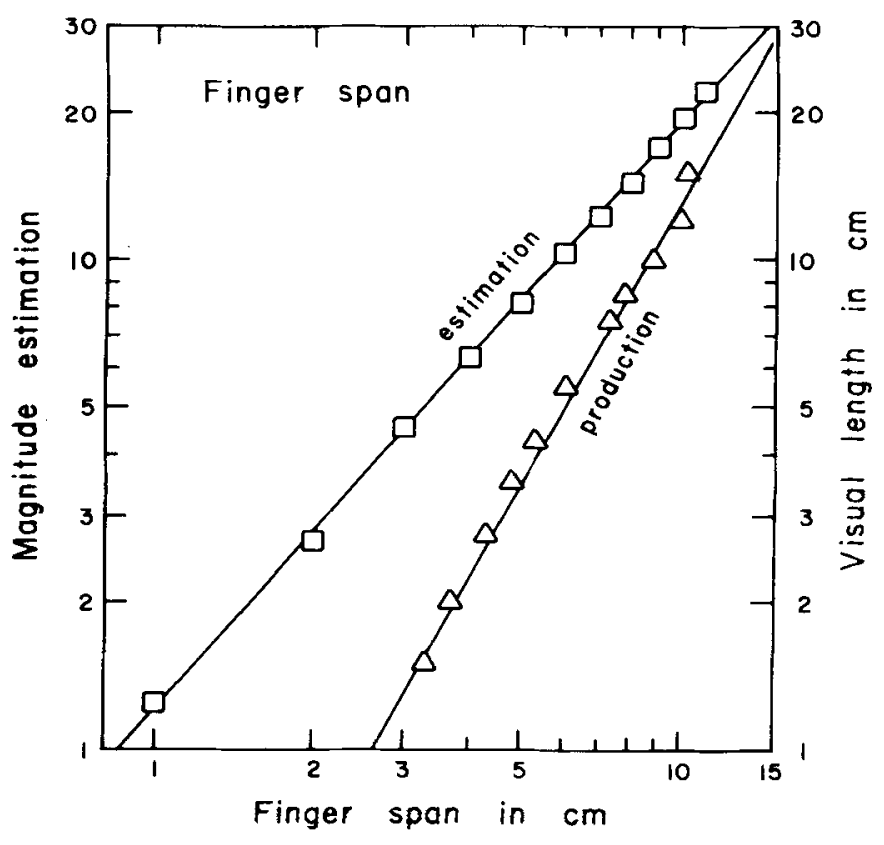

Fig. 2. Regression effect in judgments involving finger span. The squares show the results for magnitude estimation, the same function shown in Fig. 1. The triangles show the function obtained when the $\mathbf{O}$ produced various finger spans in order to match the apparent length of lines presented visually. The slope (exponent) for estimation is 1.2; the exponent for production is 1.9. The regression, or centering effect, is shown by the angle between the two lines.

and Hosman, the ratios predicted from the magnitude estimations are shown in Table 2.

The data, of course, are not error-free. One source of systematic error in matching experiments is the regression effect, or centering tendency. The $O$ tends on the average to shorten the range of whichever stimulus variable he is allowed to control. Sometimes the regression effect may be small, as when number is matched to line length (magnitude estimation) or line length is matched to number (magnitude production) (Stevens \& Guirao, 1963). Sometimes the effect may be large, as has been demonstrated in numerous experiments (Stevens \& Greenbaum, 1966). In principle, the regression effect may be canceled by a balanced design in which each of the two continua serves once as the set of criterion stimuli and once as the variable to be adjusted. The two resulting slopes (exponents) can then be averaged by taking the geometric mean. In practice, however, we have no assurance that the assumed symmetry will in fact prove sufficiently symmetrical to cancel the systematic error. Each case needs to be examined on its merits.

Unfortunately the experiments by Mashhour and Hosman did not use a balanced design that would permit the amount of regression to be estimated directly. In a key instance, however, one of the experiments provided the data needed to determine the approximate form of a missing regression line. In that key instance, the $O$ adjusted his finger span to match various line lengths presented visually. The resulting function is shown in Fig. 2, together with the magnitude estimation function for finger span. The estimation of finger span gave an exponent of 1.2, whereas the production of finger span gave an exponent of 1.9. The large regression effect shown by the angle between the two lines in Fig. 2 can probably be taken at its face value, provided we are willing to assume that it would make no essential difference 
whether the $\mathrm{O}$ matched finger span to length or to number.

For the purpose of predicting the outcome of the experiments in which the $O$ adjusts his finger span in order to match his perceptions of various criterion stimuli, the production function in Fig. 2 is clearly more appropriate than the estimation function. Accordingly, revised predictions for the five experiments with finger span have been entered in Table 2. Correction for the regression effect improves the agreement between the exponents predicted and those actually obtained.

It may be assumed that one or another degree of regression occurs also in the other modalities. As already noted, the regression between estimating and producing lengths of line is usually small, so no attempt has been made to correct for it in Table 2. Judgments of duration, however, show a fairly large and consistent regression effect. Examples from Stevens and Greenbaum (1966) are shown in Fig. 3. Two kinds of experiments are illustrated. In one the time interval was marked by a $15-W$ red lamp that turned on and off; in the other the time interval was marked by a white noise. For each kind of stimulus the Os both estimated durations and produced durations. The magnitude estimations gave power functions with exponents 0.87 with noise and 0.93 with light. These values compare nicely with the value 0.91 obtained by Mashhour and Hosman. Given such good agreement, it seems plausible to assume that the regression effect shown in Fig. 3 can be used to provide improved predictions in Table 2 . Accordingly the production exponent for time duration was taken to be 1.18 , the mean of the two production values in Fig. 3. The values thereby predicted for cross-modality matches were entered in Table 2 . Here again, the predicted values are, on the average, brought closer to the obtained values.

\section{LOUDNESS PROBLEM}

A remaining problem concerns the three experiments in which the loudness of a white noise was adjusted to match apparent weight, lightness of gray, and apparent size of surface area. Here we encounter a remarkable circumstance. The discrepancies between the predicted and the obtained exponents go in the

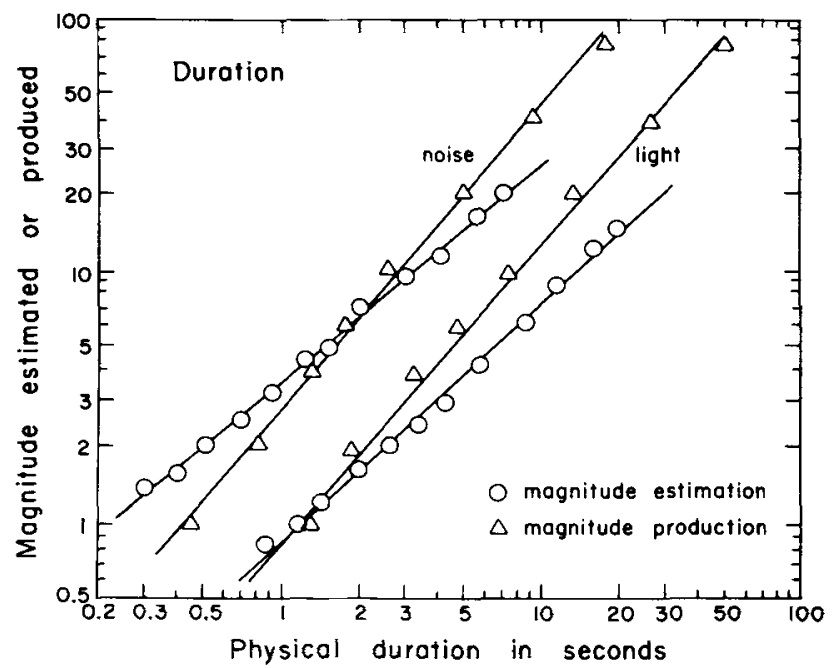

Fig. 3. Regression effect in the matching of number and duration. In one experiment the time interval was marked by a noise, in the other by a light. Circles stand for magnitude estimation, triangles for magnitude production. The data for light are shifted to the right by a factor of 3. Exponents for duration determined by noise 0.87 and 1.20 , by light 0.93 and 1.16 .

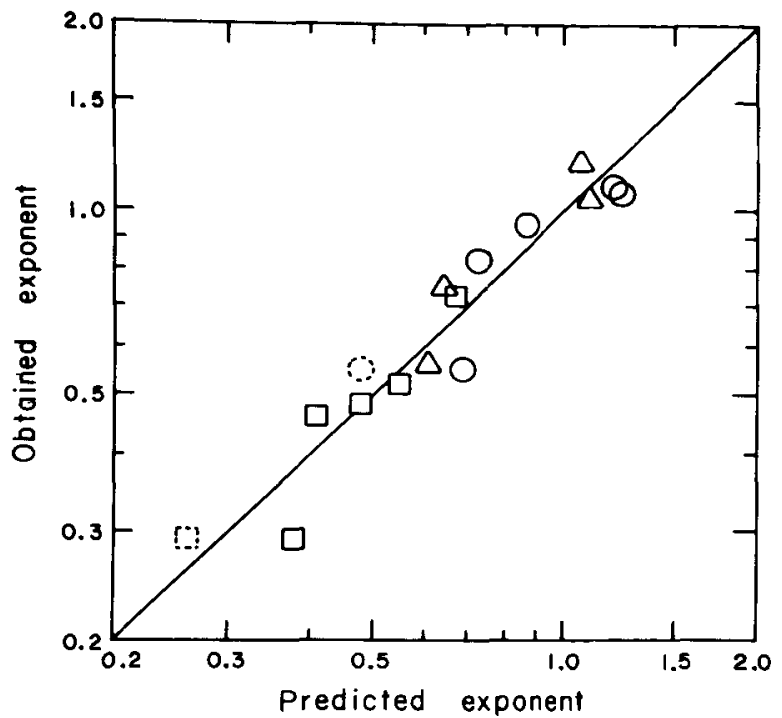

Fig. 4. Comparison between the exponents predicted by magnitude estimation and the exponents obtained in crossmodality matching experiments involving six criterion continua. The three adjusted continua were line length (shown by circles), duration (triangles), and finger span (squares). The line shows the locus of perfect coincidence between predicted and measured exponents. Root-mean-square difference between prediction and observation is 0.59 decilogs, or $14.5 \%$. Dotted symbols show the values predicted on the assumption that the matches of length and finger span were made to diameter rather than to apparent size of the circle.

wrong direction, in the sense that a correction for regression would increase the discrepancy in all three cases. The normal regression effect has become inverted. Yet that inversion is precisely what we would expect when the exponent obtained by magnitude estimation is much larger than normal, owing presumably to the short, $16.7-\mathrm{dB}$ range of stimuli used. Note that when the Os were themselves given control of the sound level, they did not restrict the stimuli to such a narrow range. Instead, they produced a range of sound energies approximately tenfold greater than the range to which the stimuli were restricted by the $\mathrm{E}$ in the experiment on magnitude estimation.

Under those circumstances, then, it would appear that a prediction based on magnitude estimation would not forecast the exponent to be expected when noise was matched to the other continua. The obtained exponent was larger than the predicted exponent, whereas we would expect, given the usual effects of regression, that the obtained exponent would be smaller than the predicted value-as was true in all cases in which finger span and time duration were matched to other continua.

As a result of the foregoing considerations, no further use will be made of the cross-modality matches involving the adjustment of the noise.

\section{TRANSITIVITY}

The data of Mashhour and Hosman provide several satisfactory corroborations of the power law, and, despite the difficulties with the noise continuum, they demonstrate the transitivity that may obtain among power-function exponents. In order to show the degree of transitivity more clearly, the values in Table 2 have been plotted in Fig. 4. The ordinate represents the values obtained in cross-modality matching. The abscissa represents the values predicted by pairs of magnitude estimation functions, 


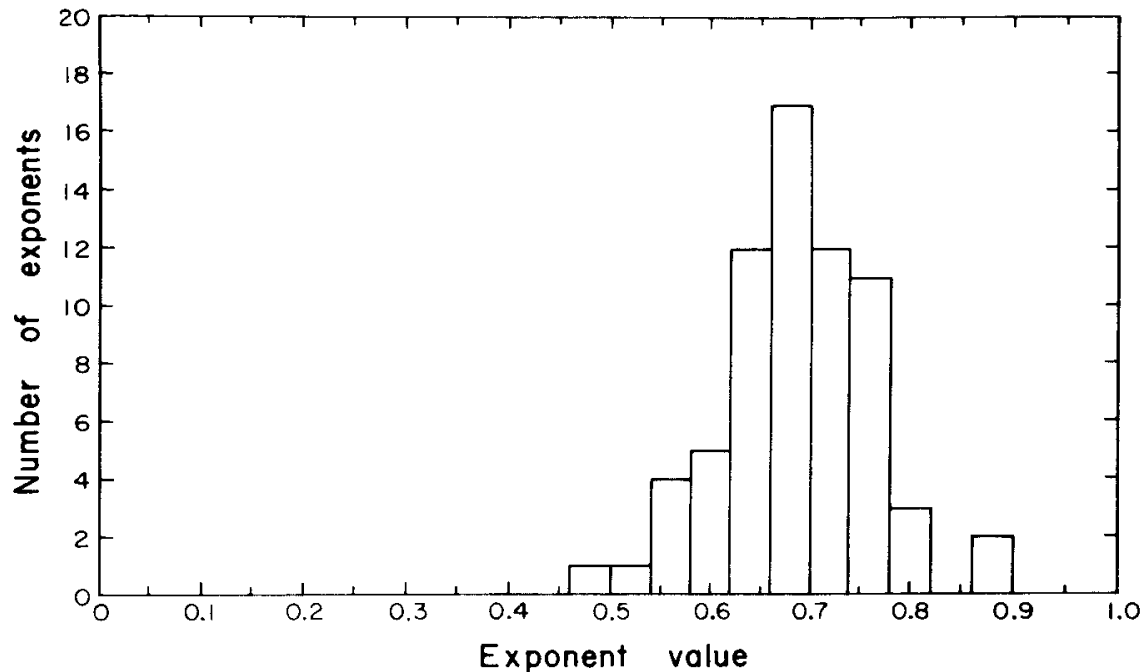

Fig. 5. Distribution of 68 estimates of the exponent of the loudness function, all based on experiments in which both number and loudness were matched to taste intensity. Both matches produced power functions. The ratio of the two exponents gives the value whose distribution is plotted. The geometric mean is 0.676 ; the standard deviation is 0.51 decilog, or $12.5 \%$. corrected for regression. The different symbols stand for the continuum that was adjusted by the $O$ : squares for finger span, triangles for time duration, and circles for line length.

If the predictions were all borne out exactly, the points would lie on the diagonal line. The 14 points cluster about the predicted line in a way to suggest that all sources of error have not been subdued. As noted above, no regression correction was made for line length, but the slight asymmetry in the positions of the circles relative to the line in Fig. 4 indicate that a small regression correction would further increase the degree of agreement.

It is of interest to compare the scatter of the points around the line in Fig. 4 with data from other experiments in which transitivity has been tested. An extensive study of the appropriate kind was carried out on taste (Moskowitz, 1968). In a total of 68 experiments, each involving 10 Os from a pool of 46 , taste intensity was scaled by two procedures: number matching (magnitude estimation) and loudness matching. The $O$ sipped a given solution of a taste substance and either assigned a number (no standard given) or, in another part of the session, adjusted the level of a noise $(500-5000 \mathrm{~Hz})$ in order to produce a loudness that matched the taste intensity. At least two matches of number and of loudness were made to each concentration of the taste substance. The four basic tastes, sweet, salt, sour, and bitter, were used. In addition, each of the taste substances was present as a background, at several different concentrations, in order to explore the masking of each taste by each other taste. The results of the 68 experiments were fitted by power functions.

A remarkable finding was that the presence of a background taste had little or no effect on the exponent of the function for the taste being judged.

Of more relevance to the present concern is the opportunity afforded for the indirect computation of the loudness exponent. Since both number and loudness were matched to the same taste continuum, the exponent to be expected if number and loudness were matched in a balanced experiment is given by the ratio of the two measured exponents. For the series of taste experiments a total of 68 exponents can be computed, a number sufficiently large to reveal the nature of the distribution to be expected in transitivity tests of this kind.

A histogram of the computed loudness exponents is shown in Fig. 5. The geometric mean of the distribution is 0.676 , which accords well with other cross-modality determinations of the exponent for noise (Stevens, 1966). As a matter of fact, the determination by way of the taste experiments by Moskowitz is perhaps the most thorough determination yet made of an exponent. The determination is especially noteworthy because, since the Os adjusted both the numbers and the loudness, we can determine the ratio between the slopes of two comparable regression lines. In that way the regression effect can be canceled to a large if not a complete extent.

Since we are here dealing with distributions of ratios, it is useful to take the logarithms of the exponents. The standard deviation of the exponents in Fig. 5 then turns out to be 0.51 decilog, which corresponds to a coefficient of variation of $12.5 \%$. That value is an empirically determined measure, not a value based on statistical assumptions. The decilog value can be compared with the scatter in Fig. 4 where the root-mean-square difference between the predicted and the obtained exponents is 0.59 decilog, or $14.5 \%$. It appears, therefore, that the scatter among the exponents in the experiments by Mashhour and Hosman was not out of line with the variability encountered in other cross-modality studies. From their studies and those of Moskowitz we obtain a potential criterion that may guide our future expectations in the domain of psychophysical power functions. In experiments comparable to the kind described, approximately two-thirds of the transitivity tests may be expected to produce exponents that agree within plus or minus half a decilog, which corresponds to a coefficient of variation of about $12 \%$.

It is of interest to note that all but one of the exponents determined by the matching functions between loudness and 10 other continua (Stevens, 1966) fall well within the distribution shown in Fig. 5 . On the basis of all 10 continua, the geometric mean of the loudness exponents turned out to be 0.67 , a value close to that determined by Moskowitz. The standard deviation of the 10 exponents was 0.69 decilog. When the case based on the felt hardness of rubber samples was omitted, the standard deviation became 0.41 decilog.

\section{THE PROBLEM OF AREA}

Thus far the only factor employed in order to refine the predictions for the cross-modality exponents of Mashhour and Hosman has been the regression effect. It should be observed, however, that the two largest discrepancies between the exponents predicted and those obtained concern the matching of a linear extent to an area. Note the last two values in the last column of Table 2 .

Martha Teghtsoonian (1965) showed that the judgment of circular area can be easily influenced by the attitude of the $O$. She showed that the $O$ can be made to judge either apparent size 
(obtained exponent 0.76 ) or physical size (obtained exponent 1.03). Most of the 10 Os stated later that they had judged physical size by judging a linear dimension of the figure and squaring it. The relevance of Teghtsoonian's work to our present problem lies in the ease with which Os may change from judging apparent size to judging a linear dimension such as diameter. It seems reasonable to expect that, when the O's task is to match a length to a circle, there may arise a strong tendency for the $\mathrm{O}$ to make the length of the line proportional to the diameter of the circle rather than to the apparent size of the circle. The same with finger span. The linear spread of the fingers may be more readily adjusted to match the diameter rather than the apparent size of the circle.

If we assume that the Os adjusted length and finger span to match apparent circle diameter, and if we further assume that apparent diameter is proportional to the square root of the area, then in Table 2 the predicted exponents would become 0.48 and 0.26 , respectively. The obtained exponents were 0.55 and 0.29 , Thus the predicted values are brought closer to the obtained values, but there is a slight overcorrection. The overcorrection of the predicted values suggests that the two assumptions made above may be only partly correct. The amount of the overcorrection can be seen graphically by the two symbols given by dotted outline in Fig. 4. Both dotted symbols lie to the left of the diagonal line, but it is interesting that they lie closer to the predicted line than do the uncorrected values. When the two corrected values are used, the root-mean-square deviation between the obtained and the predicted values in Fig. 4 is reduced from 0.59 decilog to 0.47 decilog.

\section{REFERENCES}

MARKS, L. E., \& STEVENS, J. C. The form of the psychophysical function near threshold. Perception \& Psychophysics, 1968, 4, 315-318.
MASHHOUR, M., \& HOSMAN, J. On the new "psychophysical law": A validation study. Perception \& Psychophysics, 1968a, 3, 367-375.

MASHHOUR, M., \& HOSMAN, J. On Stevens's 'psychophysical law': A validation study. Reports from the Psychological Laboratory, University of Stockholm, $1968 \mathrm{~b}$.

MOSKOWITZ, H. R. Scalcs of intensity for single and compound tastes. PhD thesis, Harvard University, 1969.

STEVENS, J. C., \& STEVENS, S. S. Brightness function: Effects of adaptation. Journal of the Optical Society of America, 1963, 53, 375-385.

STEVENS, S. S. The direct estimation of sensory magnitudes-loudness. American Journal of Psychology, 1956, 69, 1-25.

STEVENS, S. S. Matching functions between loudness and ten other continua. Perception \& Psychophysics, 1966, 1, 5.8.

STEVENS, S. S., \& E. H. GALANTER. Ratio scales and category scales for a dozen perceptual continua. Journal of Experimental Psychology, $1957,54,377-411$.

STEVENS, S. S., \& GREENBAUM, H. B. Regression effect in psychophysical judgments. Perception \& Psychophysics, 1966, 1 , 439-446.

STEVENS, S. S., \& GUIRAO, M. Subjective scaling of length and area and the matching of length to loudness and brightness. Journal of Experimental Psychology, 1963, 66, 177-186.

STEVENS, S. S., \& STEVENS, J. C. The dynamics of visual brigh tness. Laboratory of Psychophysics, Harvard University, Report PPR-246, 1960.

TEGHTSOONIAN, M. The judgment of size. American Joumal of Psy chology, 1965, 78, 392-402.

TORGERSON, W. S. Quantitative judgment scales. In H. Gulliksen and S. Messick (Eds.), Psychological scaling. New York: Wiley, 1960.

\section{NOTES}

1. Research supported by Grant NB-02974 from the National Institutes of Health (Laboratory of Psychophysics Report PPR-347-125).

2. Address: Laboratory of Psychophysics, William James Hall, Harvard University, Cambridge, Massachusetts 02138.

(Accepted for publication March 13, 1969.) 